\title{
DERMITE DAS PÁLPEBRAS - ESTUDO RETROSPECTIVO DE 5 ANOS (2009-2013)
}

\author{
Pedro Vale-Fernandes' ${ }^{1}$ Cristina Amaro², Jorge Cardoso 3 \\ 'Interno da Formação específica de Dermatologia e Venereologia/Resident, Dermatology and Venereology \\ ${ }^{2}$ Assistente Hospitalar de Dermatologia e Venereologia/Consultant, Dermatology and Venereology \\ ${ }^{3}$ Assistente Hospitalar Graduado Sénior de Dermatologia e Venereologia/Senior Graduated Consultant, Dermatology \\ and Venereology \\ Serviço de Dermatologia e Venereologia, Centro Hospitalar de Lisboa Central, Hospital Curry Cabral, Lisboa, Portugal
}

RESUMO - Introdução: A dermite das pálpebras é uma dermatose comum cuja etiologia é muitas vezes difícil de determinar, embora o eczema de contacto alérgico constitua a etiologia mais comum em doentes submetidos a provas epicutâneas. Este é um estudo retrospectivo de 5 anos para avaliar as etiologias e os alérgenos mais frequentes em doentes com eczema de contacto alérgico das pálpebras. Materiais e Métodos: Identificaram-se todos os doentes com dermite das pálpebras submetidos a provas epicutâneas na Consulta de Dermatologia do Hospital Curry Cabral no período de 2009-2013 (5 anos) por meio de uma base de dados informatizada. Resultados: No período de 5 anos (2009-2013) foi testado um total de 1341 doentes, dos quais 117 (8,7\%) foram testados por dermite das pálpebras. A etiologia mais frequente foi o eczema de contacto alérgico (54 doentes) seguida de eczema atópico (22 doentes). A resina Formoltolvenosulfonamida foi o alérgeno mais frequentemente identificado. $\bigcirc$ grupo de alérgenos mais frequentemente apurado foi o dos conservantes, seguido dos grupos das fragrâncias e fármacos.

PALAVRAS-CHAVE - Alérgenos; Dermite; Dermatite alérgica de contacto; Doenças das pálpebras; Provas epicutâneas.

\section{EYELID DERMATITIS - 5-YEAR RETROSPECTIVE STUDY (2009-2013)}

ABSTRACT - Background: Eyelid dermatitis is a common disease and its etiology frequently difficult to ascertain, although allergic contact dermatitis is the most common cause in patch-tested patients. This is a 5-year retrospective study to evaluate the most frequent causes and allergens in patients with allergic eyelid dermatitis. Materials and Methods: Using a computerized database, patients with eyelid dermatitis patch tested over a 5-year period from 2009-2013 on the Dermatology Consultation of Hospital Curry Cabral were identified. Results: Over the 5-year period (2009-2013) from a total of 1341 patch tested patients, 117 (8.7\%) had eyelid dermatitis. The most frequent etiology was allergic eyelid dermatitis (54 patients), followed by atopic dermatitis (22 patients). Tolvenesulfonamide Formaldehyde resin was the most frequent allergen identified. The preservative allergens group was the most frequently identified, followed by fragrances and drugs.

KEY-WORDS - Allergens; Dermatitis; Dermatitis, Allergic contact; Eyelid diseases; Patch tests.

\footnotetext{
Conflitos de interesse: Os autores declaram não possuir conflitos de interesse.

No conflicts of interest.

Suporte financeiro: $O$ presente trabalho não foi suportado por nenhum subsídio ou bolsa.

No sponsorship or scholarship granted.

Direito à privacidade e consentimento escrito / Privacy policy and informed consent: Os autores declaram
} 


\section{GPDEC}

que pediram consentimento ao doente para usar as imagens no artigo. The authors declare that the patient gave written informed consent for the use of its photos in this article.

Recebido/Received - Setembro/September 2013; Aceite/Accepted - Outubro/October 2014

Por decisão dos autores, este artigo não foi redigido de acordo com os termos do novo Acordo Ortográfico.

\section{Correspondênciò: \\ Dr. Pedro Vale-Fernandes}

Serviço de Dermatologia e Venereologia

Hospital de Curry Cabral - Centro Hospitalar de Lisboa Central

Rua da Beneficência, n요

1069-166 Lisboa, Portugal

\section{INTRODUÇÃO}

A dermite das pálpebras é uma dermatose comum cuja etiologia é muitas vezes difícil de determinar'. Constituem causas potenciais o eczema de contacto alérgico, eczema de contacto irritativo, eczema atópico, eczema seborreico, psoríase, rosácea e dermatomiosite, entre outras ${ }^{2}$.

O eczema de contacto alérgico é a causa mais comum de dermite das pálpebras, o que se poderá dever à espessura da pele extremamente fina nesta localização, o que facilita a penetração de potenciais alergenos ${ }^{3}$.

Modos de transferência de alérgenos incluem a aplicação intencional de medicamentos tópicos ou cosméticos, exposição a partículas ou gotículas da atmosfera, transferência de alérgenos de outras localizações anatómicas pelas mãos ou transferência sistémica ${ }^{4}$.

Para determinar o espectro actual de alérgenos associados ao eczema de contacto alérgico das pálpebras efectuou-se uma análise retrospectiva dos doentes submetidos a provas epicutâneas por dermite das pálpebras num período de 5 anos.

\section{MÉTODOS}

Identificaram-se todos os doentes submetidos a provas epicutâneas na Consulta de Dermatologia do Hospital Curry Cabral no período de 2009-2013 (5 anos) por meio de uma base de dados informatizada, registando-se também as características demográficas e os antecedentes pessoais destes doentes.

A série básica do Grupo Português de Estudo das Dermites de Contacto foi sistematicamente testada, acrescentando-se Séries Complementares e/ou produtos pessoais ou ocupacionais quando clinicamente relevante. A Metilisotiazolinona foi testada quando relevante entre 2010 e 2012.

As leituras foram efectuadas em D2 e D4 e a sua relevância atribuída na última consulta como actual, passada ou indeterminada.

\section{RESULTADOS}

No período de 5 anos (2009-2013) foi testado um total de 1341 doentes, dos quais 117 (8,7\%) foram testados por dermite das pálpebras. A maioria destes doentes $(102,87,2 \%)$ era do sexo feminino e apenas $15(12,8 \%)$ do sexo masculino. As idades estavam compreendidas entre os 9 e os 85 anos e a média de idades foi 46,0 anos.

A etiologia (Fig. 1) mais frequente foi o eczema de contacto alérgico (54 doentes) seguida de eczema

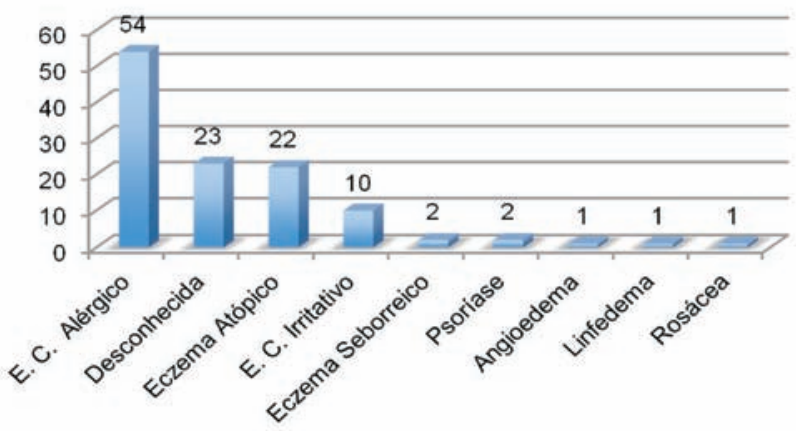

Fig 1 - Etiologia da dermite das pálpebras; E.C. - Eczema de Contacto. 


\section{GPDEC}

atópico (22 doentes). Não foi possível determinar uma etiologia em 23 doentes.

Dos 54 doentes com eczema de contacto alérgico $47(87,0 \%)$ eram do sexo feminino e $7(13,0 \%)$ do sexo masculino, com idades compreendidas entre os 19 e os 85 anos e uma média de idades de 48,5 anos.

Cinco doentes tiveram mais do que 1 reacção positiva considerada relevante, perfazendo um total de 64 positividades.
A resina ormoltolvenosulfonamida foi o alérgeno mais frequentemente identificado, com relevância actual para o uso de verniz ungueal em 9 doentes (Tabela 1).

O grupo dos conservantes foi o mais frequentemente apurado, com relevância actual em 18 doentes, seguindo-se dos grupos das fragrâncias e fármacos, identificados em 11 e 10 doentes, respectivamente (Fig. 2).

Tabela 1 - Alérgenos identificados com relevância actual. RFTS: Resina Formoltolvenosulfonamida; Kathon $\mathrm{CG}^{\circledR}$ : Metilisotiazolinona/Metilcloroisotiazolinona.

\begin{tabular}{|c|c|c|}
\hline ALERGENO & $\begin{array}{l}\text { NÚMERO DE DOENTES } \\
(\mathbf{N}=54), \mathbf{N}(\%)\end{array}$ & DESCRIÇÃO \\
\hline RFTS & $9(16,7)$ & Resina \\
\hline Kathon CG ${ }^{\circledast}$ & $8(14,8)$ & Conservante \\
\hline Metilisotiazolinona (200ppm) & $5(9,3)$ & Conservante \\
\hline Parafenilenodiamina & $4(7,4)$ & Tinta \\
\hline Metabissulfito de Sódio & $3(5,6)$ & Conservante \\
\hline Diacrilato & $2(3,7)$ & Monómero \\
\hline Aldeído Cinâmico & $2(3,7)$ & Fragrância \\
\hline Neomicina & $2(3,7)$ & Fármaco \\
\hline Mistura de Tiurans & $2(3,7)$ & Acelerador da borracha \\
\hline Álcool Cinâmico & $1(1,9)$ & Fragrância \\
\hline Azitromicina & $1(1,9)$ & Fármaco \\
\hline Bálsamo do Peru & $1(1,9)$ & Fragrância \\
\hline Benzocaína & $1(1,9)$ & Fármaco \\
\hline Budesonido & $1(1,9)$ & Fármaco \\
\hline Butirato de Hidrocortisona & $1(1,9)$ & Fármaco \\
\hline Coumarina & $1(1,9)$ & Fragrância \\
\hline Oak Moss & $1(1,9)$ & Fragrância \\
\hline Resina epóxida & $1(1,9)$ & Resina \\
\hline Serradura & $1(1,9)$ & Madeira \\
\hline
\end{tabular}




\section{GPDEC}

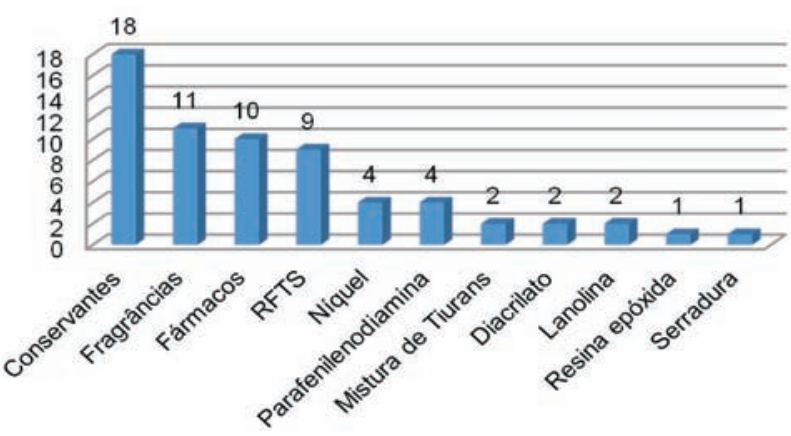

Fig 2 - Alérgenos identificados com relevância actual. RFTS: Resina Formoltolvenosulfonamida.

No grupo dos conservantes o alergeno mais frequentemente identificado foi a Metilisotiazolinona/ Metilcloroisotiazolinona, com relevância para uso de cosméticos e/ou produtos de limpeza em 8 doentes. A Metilisotiazolinona [200ppm $(0,02 \%$ aq)] teve relevância em 5 doentes (em 3 de forma isolada relativamente ao Kathon $\mathrm{CG}^{\circledR}$ ). O Metabissulfito de Sódio teve relevância para uso de cosméticos em 2 doentes e para a aplicação de gotas oftálmicas em 1 doente. A Diazolidinilureia e a Imidazolidinilureia tiveram relevância para uso de cosméticos em 1 doente cada.

No grupo das fragrâncias o Liral foi o alergeno mais frequentemente identificado, com relevância para uso de cosméticos e/ou produtos de limpeza em 4 doentes, seguido do Aldeído Cinâmico, com relevância para uso de cosméticos em 2 doentes. Outros alergenos identificados foram o Bálsamo do Peru, Hidroxicitronelal, Aldeído Cinâmico, Coumarina e Oak Moss, todos com relevância para uso de cosméticos em 1 doente cada.

No grupo dos fármacos a Neomicina teve relevância para aplicação de gotas oftálmicas em 2 doentes. A Dibucaína e a Benzocaína foram identificadas com relevância para uso de colutórios, com sensibilização por via sistémica em 2 e 1 doente, respectivamente. $\bigcirc$ Maleato de Timolol, a Azitromicina e o Trusopt solução oftálmica (Dorsolamida) tiveram relevância para aplicação de gotas oftálmicas em 1 doente cada. Neste último caso não se pôde apurar definitivamente a sensibilização à Dorsolamida por inexistência do componente puro, embora tivesse sido excluída reactividade aos excipientes da solução. O Budesonido foi identificado com relevância para uso de inalador, com sensibilização conubial por via aerotransportada em 1 doente e o Butirato de Hidrocortisona apurado com relevância para aplicação de tópicos em 1 doente.

O Níquel foi relevante em 4 doentes, dos quais $2 \mathrm{com}$ sensibilização profissional (manuseamento de moedas por bancária e lojista) e 2 por uso de cosméticos.

A Parafenilenodiamina teve relevância para uso de cosméticos, nomeadamente aplicação de tinta capilar em 4 doentes. O Diacrilato foi identificado com relevância para uso de unhas de gel em 2 doentes. A Lanolina teve relevância em 2 doentes, para o uso de cosméticos.

A Mistura de Tiurans teve relevância profissional para uso de óculos protectores em 1 doente. A Resina Epóxida e a Serradura tiveram relevância ocupacional, por via aerotransportada, em 1 doente cada.

\section{DISCUSSÃO}

A prevalência de doentes com dermite das pálpebras em doentes submetidos a provas epicutâneas é muito variável (3 a 21\%), dependendo provavelmente das séries de alérgenos testados, de composições distintas dos produtos e níveis sensibilização em diferentes áreas geográficas e padrões de referenciação ou especialização dos diferentes centros ${ }^{1,2,5-7}$.

De modo semelhante a estudos prévios ${ }^{1-3,5}$, o eczema de contacto alérgico foi a causa mais frequente de dermite das pálpebras (46\%). Resultados comparáveis foram apresentados por Landeck $L$ et a ${ }^{3}$ que avaliaram 4779 doentes submetidos a provas epicutâneas por dermite das pálpebras e relataram que $32 \%$ tinham eczema de contacto alérgico.

O predomínio de doentes do sexo feminino no grupo de doentes com eczema de contacto alérgico é conhecido e provavelmente secundário ao maior uso de cosméticos neste género.

A relação causal entre o uso de verniz ungueal e eczema de contacto alérgico das pálpebras está bem estabelecida ${ }^{8}$, embora no nosso estudo a prevalência da resina Formoltolvenosulfonamida $(16,7 \%)$ seja superior à descrita em estudos anteriores $(6,5-10 \%)^{2,9}$.

Os grupos dos conservantes e fragrâncias foram globalmente os mais frequentes, de modo semelhante ao relatado em estudos anteriores ${ }^{2,3}$ e que provavelmente reflecte a sua ampla utilização na indústria actualmente.

\section{BIBLIOGRAFIA}

1. Guin J. Eyelid dermatitis: experience in 203 cases. J Am Acad Dermatol. 2002; 47(5):755-65.

2. Amin KA, Belsito DV. aetiology of eyelid dermatitis: a 10-year retrospective analysis. Contact 
Dermatitis. 2006; 55(5):280-5.

3. Landeck L, John SM, Geier J.riorbital dermatitis in 4779 patients - patch test results during a 10-year period. Contact Dermatitis. 2014; 70(4):205-12

4. Goossens A. Contact allergic reactions on the eyes and eyelids. Bull Soc Belge Ophtalmol 2004; 292:11-7.

5. Valsecchi R, Imberti G, Martino D, Cainelli T. Eyelid dermatitis: an evaluation of 150 patients. Contact Dermatitis 1992; 27:143-7.

6. Shah M, Lewis F M, Gawkrodger D J. Facial dermatitis and eyelid dermatitis: a comparison of patch test results and final diagnoses. Contact
Dermatitis. 1996; 34:140-1.

7. Landeck L, Schalock P C, Baden L A, Gonzalez E. Periorbital contact sensitization. Am J Ophthalmol. 2010; 150:366-70.

8. Cronin E. Nail varnish. Contact dermatitis. $1^{\text {st }}$ edition. Edinburgh: Churchill Livingstone; 1980.Guin J. Eyelid dermatitis: a report of 215 patients. Contact Dermatitis 2004; 50:87-90.

9. Guin J. Eyelid dermatitis: a report of 215 patients. Contact Dermatitis 2004; 50:87-90.

10. Cooper SM. Eyelid dermatitis: an evaluation of 232 patch test patients over 5 years. Contact Dermatitis. 2000; 42(5):291-3. 